\title{
Computation of Topological Indices of NEPS of Graphs
}

\author{
Muhammad Imran $\mathbb{D}^{1},{ }^{1}$ Shehnaz Akhter, ${ }^{2}$ and Muhammad Kamran Jamil $\mathbb{D D}^{3}$ \\ ${ }^{1}$ Department of Mathematical Sciences, College of Sciences, United Arab Emirates University, Al Ain, UAE \\ ${ }^{2}$ School of Natural Sciences, National University of Sciences and Technology, Islamabad, Pakistan \\ ${ }^{3}$ Department of Mathematics, Riphah Institute of Computing and Applied Sciences, Riphah International University, Lahore, \\ Pakistan
}

Correspondence should be addressed to Muhammad Imran; imrandhab@gmail.com

Received 3 April 2021; Accepted 2 June 2021; Published 14 June 2021

Academic Editor: Muhammad Javaid

Copyright (c) 2021 Muhammad Imran et al. This is an open access article distributed under the Creative Commons Attribution License, which permits unrestricted use, distribution, and reproduction in any medium, provided the original work is properly cited.

\begin{abstract}
The inspection of the networks and graphs through structural properties is a broad research topic with developing significance. One of the methods in analyzing structural properties is obtaining quantitative measures that encode data of the whole network by a real quantity. A large quantity of graph-associated numerical invariants has been used to examine the whole structure of networks. In this analysis, degree-related topological indices have a significant place in nanotechnology and theoretical chemistry. Thereby, the computation of indices is one of the successful branches of research. The noncomplete extended $p$-sum (NEPS) of graphs is a famous general graph product. In this paper, we investigated the exact formulas of general zeroth-order Randić, Randić, and the first multiplicative Zagreb indices for NEPS of graphs.
\end{abstract}

\section{Introduction}

Let $\mathscr{H}$ be a graph and $V(\mathscr{H})$ and $E(\mathscr{H})$ be the vertex and edge sets of $\mathscr{H}$, respectively. $|V(\mathscr{H})|$ denotes the number of vertices in $\mathscr{H}$, which is known as the order of $\mathscr{H}$, and the number of elements in $E(\mathscr{H})$ is said to be the size of $\mathscr{H}$, presented by $|E(\mathscr{H})|$. The degree of a vertex $v$, presented as $d_{\mathscr{H}}(v)$, in $\mathscr{H}$ is the cardinality of linked edges with $v$. Graphs considered in this article are simple, finite, connected, and undirected. Graph theoretical notations which are not interpreted here can be seen in [1].

Topological invariants have developed a lot of applications in drug discovery, including physicochemical, modeling information of molecules, toxicology, biology, and chemistry. There are numerous graphical invariants, which are effective in chemistry and nanotechnology. Thereby, the calculation of topological invariants is one of the worthwhile domains of graphical research. Suppose that $\mathscr{T}$ corresponds to the set of all simple graphs and a function $\mathscr{F}: \mathscr{T} \longrightarrow \mathscr{R}^{+}$ is described as a topological invariant if for any set of two isomorphic $\mathscr{H}_{1}$ and $\mathscr{H}_{2}$ graphs, we have $\mathscr{F}\left(\mathscr{H}_{1}\right)=\mathscr{F}\left(\mathscr{H}_{2}\right)$. Hundreds of topological indices based on degree or distances have been introduced, but few of them gain popularity due to their high predictive power for various properties such as boiling point, density, refractive index, and molecular weight. For elaborated discussions on these invariants, we refer the reader to [2-11].

Wiener proposed the first distance-related topological invariant, named the Wiener index, and is specified as [12]

$$
\mathscr{W}(\mathscr{H})=\sum_{\left\{u^{\prime}, v^{\prime}\right\} \subseteq V(\mathscr{H})} d_{\mathscr{H}}\left(u^{\prime}, v^{\prime}\right),
$$

where $d_{\mathscr{H}}\left(u^{\prime}, v^{\prime}\right)$ is the shortest distance among $u^{\prime}$ and $v^{\prime}$ in $\mathscr{H}$. The authors in $[13,14]$ investigated the Wiener index of several graph operations.

For a graph $\mathscr{H}$, Bollobás and Erdos [15] introduced the general Randić index as

$$
R_{\gamma}(\mathscr{H})=\sum_{u^{\prime} v^{\prime} \in E(\mathscr{H})}\left(d_{\mathscr{H}}\left(u^{\prime}\right) d_{\mathscr{H}}\left(v^{\prime}\right)\right)^{\gamma},
$$

where $\gamma \in \mathscr{R}$. The general Randić index is one of the most eye-catching topological indexes in QSAR and QSPR studies [16]. There is massive research related to the mathematical 
aspects of the general Randić index $[10,17]$. Then, $R_{1 / 2}(\mathscr{H})$ is the classical Randić index [18]. Zeroth-order general Randić index for $\mathscr{H}$ is a modification of the Randić index $[19,20]$. In 2005, Li and Zheng [21] presented the zeroth-order general Randić index for graphs, which is specified as

$$
{ }^{0} R_{\gamma}(\mathscr{H})=\sum_{v^{\prime} \in V(\mathscr{H})} d_{\mathscr{H}}\left(v^{\prime}\right)^{\gamma},
$$

where $\gamma \in \mathscr{R}$.

The first $M_{1}$ and second $M_{2}$ Zagreb indices are very famous topological descriptors with several implementations in QSPR/QSAR. The first Zagreb index can be obtained by putting $\gamma=2$ in (3), while the second Zagreb index can be achieved by replacing $\gamma=1$ in (2) [22]. The chemical and mathematical aspects of the zeroth-order general Randic index have been investigated in [23-27].

Recently, the authors in [28] proposed the general multiplicative Zagreb indices for $\mathscr{H}$. The first and the second general multiplicative Zagreb indices are described:

$$
\prod_{1, \gamma}(\mathscr{H})=\prod_{u^{\prime} \in V(\mathscr{H})} d_{\mathscr{H}}\left(u^{\prime}\right)^{\gamma}, \prod_{2, \gamma}(\mathscr{H})=\prod_{u^{\prime} \in V(\mathscr{H})} d_{\mathscr{H}}\left(u^{\prime}\right)^{\gamma d_{\mathscr{H}}\left(u^{\prime}\right)},
$$

where $\gamma \in \mathscr{R}$.

These are the generalizations of the classic first and second multiplicative Zagreb indices [29]. Khalifeh et al. [30] investigated the formulas of the Zagreb indices of graph products such as Cartesian product, composition, join, and disjunction. In [31], the authors investigated the bounds for multiplicative Zagreb indices of graph operations including the join, corona product, Cartesian product, composition, and disjunction.

For graphs $\mathscr{H}_{1}, \mathscr{H}_{2}, \ldots, \mathscr{H}_{n}$ and a given set $\mathscr{B} \subseteq\{0,1\}^{n}$, the noncomplete extended $p$-sum (NEPS) of graph $\mathscr{H}_{l}$, $1 \leq l \leq n$, with respect to $\mathscr{B}$ has the vertex set and the Cartesian product of $V\left(\mathscr{H}_{l}\right), 1 \leq l \leq n$, i.e., if $\mathscr{H}=$ NEPS $\left(\mathscr{H}_{1}, \mathscr{H}_{2}, \ldots, \mathscr{H}_{n} ; \mathscr{B}\right)$ is NEPS of $\mathscr{H}_{1}, \mathscr{H}_{2}, \ldots, \mathscr{H}_{n}$, then $V(\mathscr{H})=V\left(\mathscr{H}_{1}\right) \times V\left(\mathscr{H}_{2}\right) \times \cdots \times V\left(\mathscr{H}_{n}\right)$. For $u=\left(u_{1}\right.$, $\left.\ldots, u_{n}\right), \quad v=\left(v_{1}, \ldots, v_{n}\right) \in V(\mathscr{H})$, there is an edge $\left.\left(u_{1}, \ldots, u_{n}\right)\right)\left(v_{1}, \ldots, v_{n}\right) \in E(\mathscr{H})$ if and only if there are some $n$-tuple $\left(b_{1}, \ldots, b_{n}\right) \in \mathscr{B}$ such that $u_{l}=v_{l}$ for $b_{l}=0, u_{l}$ and $v_{l}$ are distinct, and $u_{i} v_{l} \in E\left(\mathscr{H}_{l}\right)$ for $b_{l}=1$. In particular, $\operatorname{NEPS}\left(\mathscr{H}_{1} ;\{(1)\}\right)=\mathscr{H}_{1}$, and $\operatorname{NEPS}\left(\mathscr{H}_{1} ;\{\phi\}\right)=\operatorname{NEPS}\left(\mathscr{H}_{1} ;\{(0)\}\right)$ is the graph without any edge on the vertices of $\mathscr{H}_{1}$.

The NEPS operations generalize several known graph products, the Cartesian product of graphs can be acquired by taking $\mathscr{B}=\left\{b_{1}, \ldots, b_{n}\right\}$, where $b_{i}$ is an $n$-dimensional vector in which 1 is placed at the $i$ th coordinate and 0 is placed elsewhere, and the tensor product of graphs can be achieved for $\mathscr{B}=\{(1, \ldots, 1)\}$.
In 2012, Stevanoić [32] investigated the Zagreb indices for NEPS of graphs and showed that inequalities in conjecture $M_{1}(\mathscr{H}) /|V| \leq M_{2}(\mathscr{H}) /|E|$ unchanged under NEPS of graphs. In [33], the authors determined that the family of gcd-graphs and the family of NEPS of complete graphs coincide. For a more detailed study of NEPS and related topics, we refer the reader to [34-41]. In this paper, we extended the results of Stevanoic [32] and investigated the formulas of well-known degree-related topological descriptors for the NEPS of graphs.

\section{Discussion and Main Results}

We start this section with two well-known inequalities which will be beneficial in the proof of the main results.

Lemma 1. Let $g_{1}, g_{2}, \ldots, g_{n}$ be nonnegative quantities. Then,

$$
\frac{g_{1}+g_{2}+\cdots+g_{n}}{n} \geq \sqrt[n]{g_{1} g_{2}, \ldots, g_{n}}
$$

and the equality holds if and only if all $g_{k}$ are equal, $1 \leq k \leq n$.

Lemma 2. Let $g_{1}, g_{2}, \ldots, g_{n}$ be nonnegative quantities and $t_{1}, t_{2}, \ldots, t_{n}$ be nonnegative weights. Set $t=t_{1}+t_{2}+\cdots+t_{n}$. If $t>0$, then we have

$$
\frac{t_{1} g_{1}+t_{2} g_{2}+\cdots+t_{n} g_{n}}{t} \geq \sqrt[t]{g_{1}^{g_{1}} g_{2}^{t_{2}}, \ldots, g_{n}^{t_{n}}},
$$

and the equality holds if and only if all $g_{k}$ with $t_{k}>0$ are equal, $1 \leq k \leq n$.

Lemma 3 (see [42]). Let $\mathscr{H}=\operatorname{NEPS}\left(\mathscr{H}_{1}, \ldots, \mathscr{H}_{n} ; \mathscr{B}\right)$; then, the degree of a vertex $u=\left(u_{1}, u_{2}, \ldots, u_{n}\right)$ is given as

$$
d_{\mathscr{H}}(u)=\sum_{\beta \in \mathscr{B}} \prod_{i=1}^{n} d_{\mathscr{H}_{i}}^{\beta_{i}}\left(u_{i}\right)
$$

Next, we derive the expression of the zeroth-order general Randić index of the NEPS of graphs for $\gamma \in \mathbb{Z}^{+}$.

Theorem 1. Let $\mathscr{H}=\operatorname{NEPS}\left(\mathscr{H}_{1}, \mathscr{H}_{2}, \ldots, \mathscr{H}_{n} ; \mathscr{B}\right)$, where for $l=1, \ldots, n, n \geq 2, \mathscr{H}_{l}=\left(V_{l}, E_{l}\right)$. If $\gamma \in \mathbb{Z}^{+}$, then

$$
{ }^{0} R_{\gamma}(\mathscr{H})=\sum_{\left(\beta^{1}, \ldots, \beta^{\gamma}\right) \in \mathscr{B}^{\gamma}} \prod_{l=1}^{n} R_{\left(\beta_{l}^{1}+\cdots+\beta_{l}^{\gamma}\right)}\left(\mathscr{H}_{l}\right) .
$$

Proof. Let $\mathscr{H}$ be the NEPS graph of $\mathscr{H}_{1}, \mathscr{H}_{2}, \ldots, \mathscr{H}_{n}$. Then, by applying Lemma 3 in (3), we acquire 


$$
\begin{aligned}
& { }^{0} R_{\gamma}(\mathscr{H})=\sum_{u \in V(\mathscr{H})}\left(d_{\mathscr{H}}(u)\right)^{\gamma}=\sum_{\left(u_{1}, \ldots, u_{n}\right) \in V_{1} \times \cdots \times V_{n}}\left(\sum_{\beta \in \mathscr{B}} \prod_{l=1}^{n} d_{\mathscr{H}_{l}}^{\beta_{l}}\left(u_{l}\right)\right)^{\gamma} \\
& =\sum_{\left(u_{1}, \ldots, u_{n}\right) \in V_{1} \times \ldots \times V_{n}}\left[\left(\sum_{\beta^{1} \in \mathscr{B}} \prod_{l=1}^{n} d_{\mathscr{H}_{l}}^{\beta_{l}^{1}}\left(u_{l}\right)\right) \times\left(\sum_{\beta^{2} \in \mathscr{B}} \prod_{l=1}^{n} d_{\mathscr{H}_{l}}^{\beta_{l}^{2}}\left(u_{l}\right)\right) \times \ldots \times\left(\sum_{\beta^{\gamma} \in \mathscr{B}} \prod_{l=1}^{n} d_{\mathscr{H}_{l}}^{\beta_{l}^{\gamma}}\left(u_{l}\right)\right)\right] \\
& =\sum_{\left(u_{1}, \ldots, u_{n}\right) \in V_{1} \times \cdots \times V_{n}} \sum_{\left(\beta^{1}, \ldots, \beta^{\gamma}\right) \in \mathscr{B} \times \cdots \times \mathscr{B}} \prod_{l=1}^{n} d_{\mathscr{H}_{l}}^{\left(\beta_{l}^{1}+\cdots+\beta_{l}^{\gamma}\right)}\left(u_{l}\right) \\
& =\sum_{\left(\beta^{1}, \ldots, \beta^{\gamma}\right) \in \mathscr{B}^{\gamma}} \sum_{\left(u_{1}, \ldots, u_{n}\right) \in V_{1} \times \cdots \times V_{n}} \prod_{l=1}^{n} d_{\mathscr{H}_{l}}^{\left(\beta_{l}^{1}+\cdots+\beta_{l}^{\gamma}\right)}\left(u_{l}\right) \\
& =\sum_{\left(\beta^{1}, \ldots, \beta^{\gamma}\right) \in \mathscr{B}^{\gamma}} \prod_{l=1}^{n} \sum_{u_{l} \in V_{l}} d_{\mathscr{H}_{l}}^{\left(\beta_{l}^{1}+\cdots+\beta_{l}^{\gamma}\right)}\left(u_{l}\right) \\
& =\sum_{\left(\beta^{1}, \ldots, \beta^{\gamma}\right) \in \mathscr{B}^{\gamma}} \prod_{l=1}^{n}{ }^{0} R_{\left(\beta_{l}^{1}+\cdots+\beta_{l}^{\gamma}\right)}\left(u_{l}\right) \text {, }
\end{aligned}
$$

which is the required result.

For $\gamma=2$, the zeroth-order general Randić index gives the famous first Zagreb index, and we have the following corollary.

Corollary 1 (see [32]). Let $\mathscr{H}=\operatorname{NEPS}\left(\mathscr{H}_{1}, \mathscr{H}_{2}, \ldots, \mathscr{H}_{n}\right.$; $\mathscr{B})$, where $\mathscr{H}_{l}=\left(V_{l}, E_{l}\right)$ for $l=1, \ldots, n, n \geq 2$. Then, the first Zagreb index is

$$
{ }^{0} R_{2}(\mathscr{H})=\sum_{\left(\beta^{1}, \beta^{2}\right) \in \mathscr{B}^{2}} \prod_{l=1}^{n}{ }^{0} R_{\left(\beta_{l}^{1}+\beta_{l}^{2}\right)}\left(\mathscr{H}_{l}\right) .
$$

Corollary 2. Let $\mathscr{H}=\mathscr{H}_{1} \otimes \cdots \otimes \mathscr{H}_{n}$ be the tensor product of graphs, where $\mathscr{H}_{l}=\left(V_{l}, E_{l}\right)$ with $l=1, \ldots, n, n \geq 2$, and $\gamma \in \mathbb{Z}^{+}$. Then,

$$
{ }^{0} R_{\gamma}(\mathscr{H})=\prod_{l=1}^{n}{ }^{0} R_{\gamma}\left(\mathscr{H}_{l}\right) .
$$

We proceed further to describe an upper bound of the zeroth-order general Randic index of NEPS graphs for $\gamma \in \mathbb{Z}^{-}$.

Theorem 2. Let $\mathscr{H}=\operatorname{NEPS}\left(\mathscr{H}_{1}, \mathscr{H}_{2}, \ldots, \mathscr{H}_{n} ; \mathscr{B}\right)$, where $\mathscr{H}_{l}=\left(V_{l}, E_{l}\right)$ with $l=1, \ldots, n, n \geq 2$, and $\gamma \in \mathbb{Z}^{-}$. Then,

$$
{ }^{0} R_{\gamma}(\mathscr{H}) \geq \frac{|V|^{2}}{\sum_{\left(\beta^{1}, \ldots, \beta^{\gamma}\right) \in \mathscr{B}^{\gamma}} \prod_{l=1}^{n} R_{\left(\beta_{l}^{1}+\cdots+\beta_{l}^{\gamma}\right)}\left(\mathscr{H}_{l}\right)},
$$

and the equality holds if and only if $\mathscr{H}_{l}$ 's, $1 \leq l \leq n$, are regular graphs.

Proof. For $\gamma \in \mathbb{Z}^{-}$, using Lemma 3 in (3), we have

$$
\begin{aligned}
& { }^{0} R_{\gamma}(\mathscr{H})=\sum_{u \in V(\mathscr{H})} d_{\mathscr{H}}(u)^{\gamma}=\sum_{\left(u_{1}, \ldots, u_{n}\right) \in V_{1} \times \cdots \times V_{n}}\left(\sum_{\beta \in \mathscr{B}} \prod_{l=1}^{n} d_{\mathscr{H}_{l}}^{\beta_{l}}\left(u_{l}\right)\right)^{\gamma} \\
& =\sum_{\left(u_{1}, \ldots, u_{n}\right) \in V_{1} \times \cdots \times V_{n}}\left[\left(\frac{1}{\sum_{\beta^{1} \in \mathscr{B}} \prod_{l=1}^{n} d_{\mathscr{H}_{l}}^{\beta_{l}^{1}}\left(u_{l}\right)}\right) \times\left(\frac{1}{\sum_{\beta^{1} \in \mathscr{B}} \prod_{l=1}^{n} d_{\mathscr{H}_{l}}^{\beta_{l}^{2}}\left(u_{l}\right)}\right) \times \cdots \times\left(\frac{1}{\sum_{\beta^{1} \in \mathscr{B}} \prod_{l=1}^{n} d_{\mathscr{H}_{l}}^{\beta_{l}^{\gamma}}\left(u_{l}\right)}\right)\right] \\
& =\sum_{\left(u_{1}, \ldots, u_{n}\right) \in V_{1} \times \cdots \times V_{n}}\left(\frac{1}{\sum_{\left(\beta^{1}, \ldots, \beta^{\gamma}\right) \in \mathscr{B} \times \cdots \times \mathscr{B}} \prod_{l=1}^{n} d_{\mathscr{H}_{l}}^{\left(\beta_{l}^{\left.1+\cdots+\beta_{l}^{\gamma}\right)}\left(u_{l}\right)\right.}}\right) \\
& \geq \frac{|V|^{2}}{\sum_{\left(u_{1}, \ldots, u_{n}\right) \in V_{1} \times \cdots \times V_{n}} \sum_{\left(\beta^{1}, \ldots, \beta^{\gamma}\right) \in \mathscr{B} \times \cdots \times \mathscr{B}} \prod_{l=1}^{n} d_{\mathscr{H}_{l}}^{\left(\beta_{l}^{1}+\cdots+\beta_{l}^{\gamma}\right)}\left(u_{l}\right)}
\end{aligned}
$$

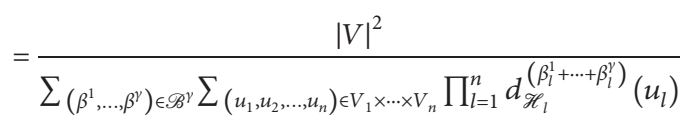

$$
\begin{aligned}
& =\frac{|V|^{2}}{\sum_{\left(\beta^{1}, \ldots, \beta^{\gamma}\right) \in \mathscr{B}^{\gamma}} \prod_{l=1}^{n} \sum_{u_{l} \in V_{l}} d_{\mathscr{H}_{l}}^{\left(\beta_{l}^{1}+\cdots+\beta_{l}^{\gamma}\right)}\left(u_{l}\right)} \\
& =\frac{|V|^{2}}{\sum_{\left(\beta^{1}, \ldots, \beta^{\gamma}\right) \in \mathscr{B}^{\gamma}} \prod_{l=1}^{n}{ }^{0} R_{\left(\beta_{l}^{1}+\cdots+\beta_{l}^{\gamma}\right)}\left(\mathscr{H}_{l}\right)},
\end{aligned}
$$


hence, the required result.

Corollary 3. Let $\mathscr{H}=\mathscr{H}_{1} \otimes \cdots \otimes \mathscr{H}_{n}$ be the tensor product of $n$ graphs, where $\mathscr{H}_{l}=\left(V_{l}, E_{l}\right)$ for $l=1,2, \ldots, n, n \geq 2$, and $\gamma \in \mathbb{Z}^{-}$. Then,

$$
{ }^{0} R_{\gamma}(\mathscr{H}) \geq \frac{\prod_{l=1}^{n}\left|V_{l}\right|^{2}}{\prod_{l=1}^{n}{ }^{0} R_{\gamma}\left(\mathscr{H}_{l}\right)},
$$

and the equality holds if and only if $\mathscr{H}_{l}, s, 1 \leq l \leq n$, are regular graphs.

In the upcoming theorem, we derive the result of NEPS graphs with respect to the general Randić index.
Theorem 3. Let $\mathscr{H}=\operatorname{NEPS}\left(\mathscr{H}_{1}, \mathscr{H}_{2}, \ldots, \mathscr{H}_{n} ; \mathscr{B}\right)$, where $\mathscr{H}_{l}=\left(V_{l}, E_{l}\right)$ for $l=1, \ldots, n, n \geq 2$. For $\gamma \in \mathbb{Z}^{+}$, the Randic index of $\mathscr{H}$ is given as

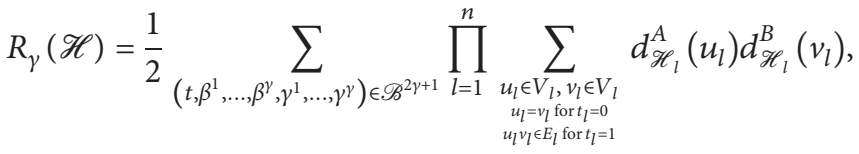

where $A=\beta_{l}^{1}+\cdots+\beta_{l}^{\gamma}, B=\gamma_{l}^{1}+\cdots+\gamma_{l}^{\gamma}$, and $0 \leq A, B \leq \gamma$.

Proof. Let $\mathscr{H}=\operatorname{NEPS}\left(\mathscr{H}_{1}, \mathscr{H}_{2}, \ldots, \mathscr{H}_{n} ; \mathscr{B}\right)$; then, the degree of each vertex is provided by Lemma 3 . Thus, the definition of the general Randić index can be described as

$$
\begin{aligned}
& R_{\gamma}(\mathscr{H})=\sum_{\{u, v\} \in E(\mathscr{H})}\left(d_{\mathscr{H}}(u) d_{\mathscr{H}}(v)\right)^{\gamma} \\
& =\frac{1}{2} \sum_{t \in \mathscr{B}} \sum_{\substack{\left(u_{1}, \ldots, u_{n}\right) \in V_{1} \times \cdots \times V_{n} \\
\left(v_{1}, \ldots, v_{n}\right) \in V_{1} \times \cdots \times V_{n} \\
u_{l}=v_{l} \text { or } t_{l}=0 \\
u_{i} l_{l} \in E_{l} \text { for } t_{l}=1}}\left[\left(\sum_{\beta \in \mathscr{B}} \prod_{l=1}^{n} d_{\mathscr{H}_{l}}^{\beta_{l}}\left(u_{l}\right)\right)\left(\sum_{\gamma \in \mathscr{B}} \prod_{l=1}^{n} d_{\mathscr{H}_{l}}^{\gamma_{l}}\left(v_{l}\right)\right)\right]^{\gamma} \\
& =\frac{1}{2} \sum_{\substack { t \in \mathscr{B} \\
\begin{subarray}{c}{\left(u_{1}, \ldots, u_{n}\right) \in V_{1} \times \ldots \times V_{n} \\
\left(v_{1}, \ldots, v_{n}\right) \in V_{1} \times \ldots \times V_{n} \\
u_{l}=v_{l} \text { fot } t_{l}=0 \\
u_{i} \nu_{l} \in E_{l} \text { for } t_{l}=1{ t \in \mathscr { B } \\
\begin{subarray} { c } { ( u _ { 1 } , \ldots , u _ { n } ) \in V _ { 1 } \times \ldots \times V _ { n } \\
( v _ { 1 } , \ldots , v _ { n } ) \in V _ { 1 } \times \ldots \times V _ { n } \\
u _ { l } = v _ { l } \text { fot } t _ { l } = 0 \\
u _ { i } \nu _ { l } \in E _ { l } \text { for } t _ { l } = 1 } }\end{subarray}}\left[\left(\sum_{\beta^{1} \in \mathscr{B}} \prod_{l=1}^{n} d_{\mathscr{H}_{l}}^{\beta_{l}^{1}}\left(u_{l}\right)\right)\left(\sum_{\gamma^{1} \in \mathscr{B}} \prod_{l=1}^{n} d_{\mathscr{H}_{l}}^{\gamma_{l}^{1}}\left(v_{l}\right)\right) \times\left(\sum_{\beta^{2} \in \mathscr{B}} \prod_{l=1}^{n} d_{\mathscr{H}_{l}}^{\beta_{l}^{2}}\left(u_{l}\right)\right)\right. \\
& \left.\left(\sum_{\gamma^{2} \in \mathscr{B}} \prod_{l=1}^{n} d_{\mathscr{H}_{l}}^{\gamma_{l}^{2}}\left(v_{l}\right)\right) \times \cdots \times\left(\sum_{\beta^{\gamma} \in \mathscr{B}} \prod_{l=1}^{n} d_{\mathscr{H}_{l}}^{\beta_{l}^{\gamma}}\left(u_{l}\right)\right)\left(\sum_{\gamma^{\gamma} \in \mathscr{B}} \prod_{l=1}^{n} d_{\mathscr{H}_{l}}^{\gamma_{l}^{\gamma}}\left(v_{l}\right)\right)\right] \\
& =\frac{1}{2} \sum_{\left(t, \beta^{1}, \ldots, \beta^{\gamma}, \gamma^{1}, \ldots, \gamma^{\gamma}\right) \in \mathscr{B}^{2 \gamma+1}} \sum_{\substack{\left(u_{1}, \ldots, u_{n}\right) \in V_{1} \times \cdots \times V_{n} \\
\left(v_{1}, \ldots, n_{n}\right) \in V_{1} \times \cdots \times V_{n} \\
u_{l}=v_{l} \text { for } t_{l}=0 \\
u_{i} v_{l} \in E_{l} \text { for } t_{l}=1}} \prod_{l=1}^{n} d_{\mathscr{H}_{l}}^{\beta_{l}^{1}+\cdots+\beta_{l}^{\gamma}}\left(u_{l}\right) d_{\mathscr{H}_{l}}^{\gamma_{l}^{1}+\cdots+\gamma_{l}^{\gamma}}\left(v_{l}\right)
\end{aligned}
$$

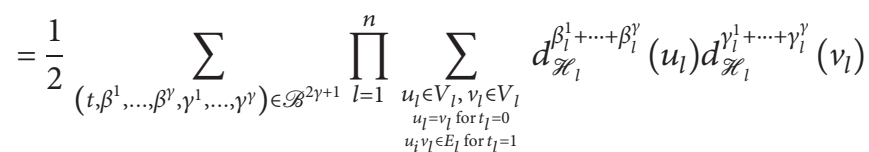

$$
\begin{aligned}
& =\frac{1}{2} \sum_{\left(t, \beta^{1}, \ldots, \beta^{\gamma}, \gamma^{1}, \ldots, \gamma^{\gamma}\right) \in \mathscr{B}^{2 \gamma+1}} \prod_{\substack{l=1 \\
l}}^{n} \sum_{\substack{u_{l} \in V_{l}, v_{l} \in V_{l} \\
u_{l}=v_{l} \text { for } t_{l}=0 \\
u_{i} v_{l} \in \ell_{l} \text { for } t_{l}=1}} d_{\mathscr{H}_{l}}^{A}\left(u_{l}\right) d_{\mathscr{H}_{l}}^{B}\left(v_{l}\right),
\end{aligned}
$$

where $A=\beta_{l}^{1}+\cdots+\beta_{l}^{\gamma}, B=\gamma_{l}^{1}+\cdots+\gamma_{l}^{\gamma}$, and $0 \leq A, B \leq \gamma$.

If $A=B=K$ for some $K \in \mathbb{Z}^{+}$, then from the above result, we obtain the following:

$$
R_{\gamma}(\mathscr{H})=\frac{1}{2} \sum_{\left(t, \beta^{1}, \ldots, \beta^{\gamma}, \gamma^{1}, \ldots, \gamma^{\gamma}\right) \in \mathscr{B}^{2 \gamma+1}} \prod_{l=1}^{n} 2 R_{K}\left(\mathscr{H}_{l}\right) .
$$

In the upcoming result, the general multiplicative Zagreb index of NEPS of graphs $\mathscr{H}_{1}, \mathscr{H}_{2}, \ldots, \mathscr{H}_{n}$ is discussed.

Theorem 4. Let $\mathscr{H}=\operatorname{NEPS}\left(\mathscr{H}_{1}, \mathscr{H}_{2}, \ldots, \mathscr{H}_{n} ; \mathscr{B}\right)$, where $\mathscr{H}_{l}=\left(V_{l}, E_{l}\right)$ for $l=1, \ldots, n$ and $n \geq 2$. If $\gamma \in \mathbb{Z}^{+}$, then 


$$
\prod_{1, \gamma}(\mathscr{H}) \leq\left(\frac{\sum_{\left(\beta^{1}, \ldots, \beta^{\nu}\right) \in \mathscr{B} \times \cdots \times \mathscr{B}} \prod_{l=1}^{n}{ }^{0} R_{\beta_{l}^{1}, \ldots, \beta_{l}^{\gamma}}\left(\mathscr{H}_{l}\right)}{|V|}\right)^{\| V \mid}
$$

and the equality holds if and only if $\mathscr{H}_{l} s, 1 \leq l \leq n$, are regular graphs.

Proof. Using Lemma 3, the definition of the general multiplicative Zagreb index for $\mathscr{H}$ can be written as

$$
\begin{aligned}
& \prod_{1, \gamma}(\mathscr{H})=\prod_{u \in V(\mathscr{H})}\left(d_{\mathscr{H}}(u)\right)^{\gamma}=\prod_{\left(u_{1}, \ldots, u_{n}\right) \in V_{1} \times \cdots \times V_{n}}\left(\sum_{\beta \in \mathscr{P}} \prod_{l=1}^{n} d_{\mathscr{H}}^{\beta_{\mathscr{H}_{l}}}\left(u_{l}\right)\right)^{\gamma} \\
& \leq\left(\frac{\sum_{\left(u_{1}, \ldots, u_{n}\right) \in V_{1} \times \cdots \times V_{n}}\left(\sum_{\beta \in \mathscr{R}} \prod_{l=1}^{n} d_{\mathscr{C}_{l}}^{\beta_{l}}\left(u_{l}\right)\right)^{\gamma}}{|V|}\right)^{|V|} \\
& =\left(\frac{\sum_{\left(u_{1}, \ldots, u_{n}\right) \in V_{1} \times \ldots \times V_{n}}\left[\left(\sum_{\beta^{1} \in \mathscr{B}} \prod_{l=1}^{n} d_{\mathscr{H}_{l}}^{\beta_{l}^{1}}\left(u_{l}\right)\right) \times\left(\sum_{\beta^{2} \in \mathscr{B}} \prod_{l=1}^{n} d_{\mathscr{H}_{l}}^{\beta_{l}^{2}}\left(u_{l}\right)\right) \times \ldots \times\left(\sum_{\beta^{\gamma} \in \mathscr{B}} \prod_{l=1}^{n} d_{\mathscr{H}_{l}}^{\beta_{l}^{\gamma}}\left(u_{l}\right)\right)\right]}{|V|}\right)^{|V|} \\
& =\left(\frac{\sum_{\left(u_{1}, \ldots, u_{n}\right) \in V_{1} \times \cdots \times V_{n}} \sum_{\left(\beta^{1}, \ldots, \beta^{\eta}\right) \in \mathscr{B} \times \cdots \times \mathscr{B}} \prod_{l=1}^{n} d_{\mathscr{H}_{l}}^{\beta_{l}^{1}+\cdots+\beta_{l}^{\gamma}}\left(u_{l}\right)}{|V|}\right)^{|V|} \\
& =\left(\frac{\sum_{\left(\beta^{1}, \ldots, \beta^{\gamma}\right) \in \mathscr{B} \times \ldots \times \mathscr{B}} \sum_{\left(u_{1}, \ldots, u_{n}\right) \in V_{1} \times \ldots \times V_{n}} \prod_{l=1}^{n} d_{\mathscr{H}_{l}}^{\beta_{l}^{1}+\ldots+\beta_{l}^{\gamma}}\left(u_{l}\right)}{|V|}\right)^{|V|}
\end{aligned}
$$

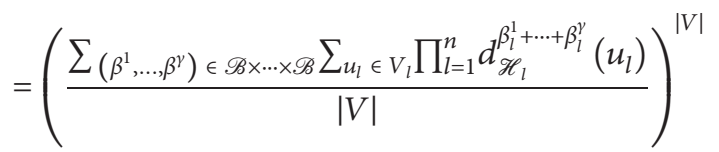

$$
\begin{aligned}
& =\left(\frac{\sum_{\left(\beta^{1}, \ldots, \beta^{\gamma}\right) \in \mathscr{B} \times \cdots \times \mathscr{B}} \prod_{l=1}^{n}{ }^{0} R_{\beta_{l}^{1}, \ldots, \beta_{l}^{\gamma}}\left(\mathscr{H}_{l}\right)}{|V|}\right)^{|V|} .
\end{aligned}
$$

This completes the proof.

\section{Conclusion}

The investigation of graphs with the help of graph invariants is a very successful method, which performs an appreciable part in predicting the physicochemical characterizations of the given graphs. Thereby, the derivations of topological invariants are an attractive, appealing, and interesting line of research in the field of graph theory. The noncomplete extended $p$-sum (NEPS) of graphs is a notable generalization of the graph product. In this paper, we have provided the results of the general zeroth-order Randić, Randić, and first multiplicative Zagreb indices for NEPS of graphs. It is more interesting to compute the eccentricity-based indices, PI, and Szeged and Mostar indices of NEPS of graphs.

\section{Data Availability}

The data used to support the findings of this study are included within the article.

\section{Conflicts of Interest}

The authors declare that there are no conflicts of interest regarding the publication of this paper.

\section{Acknowledgments}

This research was supported by the UPAR Grants of United Arab Emirates University via Grant nos. G00002590 and G00003271.

\section{References}

[1] J. A. Bondy and U. S. R. Murty, Graph Theory, Springer, Berlin, Germany, 2008.

[2] S. Akhter and M. Imran, "The sharp bounds on general sumconnectivity index of four operations on graphs," Journal of Inequalities and Applications, vol. 2016, no. 1, pp. 241-251, 2016.

[3] S. Akhter and M. Imran, "Computing the forgotten topological index of four operations on graphs," AKCE 
International Journal of Graphs and Combinatorics, vol. 14, no. 1, pp. 70-79, 2017.

[4] S. Akhter, M. Imran, W. Gao, and R. Farahani, "On topological indices of honeycomb networks and graphene networks," Hacettepe Journal of Mathematics and Statistics, vol. 47, pp. 19-35, 2018.

[5] S. Akhter, M. Imran, and Z. Raza, "Bounds for the general sum-connectivity index of composite graphs," Journal of Inequalities and Applications, vol. 76, no. 1, pp. 70-81, 2017.

[6] E. Estrada, The Structure of Complex Networks, Oxford University Press, New York, NY, USA, 2011.

[7] I. Gutman, "Degree-based topological indices," Croatica Chemica Acta, vol. 86, no. 4, pp. 351-361, 2013.

[8] M. Imran and S. Akhter, "Degree-based topological indices of double graphs and strong double graphs discrete," Mathematics, Algorithms and Applications, vol. 9, no. 5, Article ID 1750066, 2017.

[9] S. M. Hosamani, R. G. Boli, V. M. Gadag, and V. M. Gadag, "QSPR analysis of certain graph theocratical matrices and their corresponding energy," Applied Mathematics and Nonlinear Sciences, vol. 2, no. 1, pp. 131-150, 2017.

[10] R. Todeschini and V. Consonni, "New local vertex invariants and molecular descriptors based on functions of the vertex degrees," MATCH - Communications in Mathematical and in Computer, vol. 64, pp. 359-372, 2010.

[11] H. Yang, M. Imran, S. Akhter, Z. Iqbal, and M. K. Siddiqui, "On distance-based topological descriptors of subdivision vertex-edge join of three graphs," IEEE Access, vol. 7, pp. 143381-143391, 2019.

[12] H. Wiener, "Structural determination of paraffin boiling points," Journal of the American Chemical Society, vol. 69, no. 1, pp. 17-20, 1947.

[13] M. Eliasi, G. Raeisi, and B. Taeri, "Wiener index of some graph operations," Discrete Applied Mathematics, vol. 160, no. 9, pp. 1333-1344, 2012.

[14] Y.-N. Yeh and I. Gutman, "On the sum of all distances in composite graphs," Discrete Mathematics, vol. 135, no. 1-3, pp. 359-365, 1994.

[15] B. Bollobás and P. Erdös, "Graphs of extremal weights," Ars Combinatoria, vol. 50, pp. 225-233, 1998.

[16] J. Devillers and A. T. Balaban, Topological Indices and Related Descriptors in QSAR and QSPR, Gordon \& Breach, Amsterdam, Netherlands, 1999.

[17] X. Li and I. Gutman, Mathematical Aspects of Randic Type Molecular Structure Description, University of Kragujevac Rectorate, Kragujevac, Serbia, 2006.

[18] M. Randic, "Characterization of molecular branching," Journal of the American Chemical Society, vol. 97, no. 23, pp. 6609-6615, 1975.

[19] Y. Hu, X. Li, Y. Shi, and T. Xu, "Connected $(n, m)$-graphs with minimum and maximum zeroth-order general Randić index," Discrete Applied Mathematics, vol. 155, pp. 1044-1054, 2007.

[20] H. Hua and H. Deng, "On unicycle graphs with maximum and minimum zeroth-order genenal Randić index," Journal of Mathematical Chemistry, vol. 41, no. 2, pp. 173-181, 2007.

[21] X. Li and J. Zheng, "A unified approach to the extremal trees for different indices," MATCH Communications in Mathematical and in Computer Chemistry, vol. 54, pp. 195-208, 2005.

[22] I. Gutman and N. Trinajstić, "Graph theory and molecular orbitals total $\varphi$-electron energy of alternant hydrocarbons," Chemical Physics Letters, vol. 17, no. 4, pp. 535-538, 1972.
[23] A. Ali, A. A. Bhatti, and Z. Raza, "A note on the zeroth-order general randić index of cacti and polyomino chains," Iranian Journal of Mathematical Chemistry, vol. 5, pp. 143-152, 2014.

[24] H. Ahmed, A. A. Bhatti, and A. Ali, "Zeroth-order general Randić index of cactus graphs," AKCE International Journal of Graphs and Combinatorics, vol. 16, no. 2, pp. 182-189, 2019.

[25] Y. Hu, X. Li, Y. Shi, T. Xu, and I. Gutman, "On molecular graphs with smallest and largest zeroth-order general randić index," MATCH Communications in Mathematical and in Computer Chemistry, vol. 54, no. 2, pp. 425-434, 2005.

[26] S. Li and M. Zhang, "Sharp bounds on the zeroth-order general randić indices of conjugated bicyclic graphs," Mathematical and Computer Modelling, vol. 53, no. 9-10, pp. 1990-2004, 2011.

[27] G. Su, L. Xiong, X. Su, and G. Li, "Maximally edge-connected graphs and zeroth-order general Randić index for \$\$ \alpha \le $-1 \$ \$ \alpha \leq-1$," Journal of Combinatorial Optimization, vol. 31, no. 1, pp. 182-195, 2016.

[28] T. Vetrík and S. Balachandran, "General multiplicative Zagreb indices of trees," Discrete Applied Mathematics, vol. 247, pp. 341-351, 2018.

[29] R. Todeschini and V. Consonni, Handbook of Molecular Descriptors, Wiley VCH, Weinheim, Germany, 2000.

[30] M. H. Khalifeh, H. Yousefi-Azari, and A. R. Ashrafi, "The first and second Zagreb indices of some graph operations," Discrete Applied Mathematics, vol. 157, no. 4, pp. 804-811, 2009.

[31] K. C. Das, A. Yurttas, M. Togan, A. Cevik, and I. Cangul, "The multiplicative zagreb indices of graph operations," Journal of Inequalities and Applications, vol. 2013, no. 1, p. 90, 2013.

[32] D. Stevanoić, "Comparing the zagreb indices of the NEPS of graphs," Applied Mathematics and Computation, vol. 219, pp. 1082-1086, 2012.

[33] W. Klotz and T. Sander, "GCD-graphs and NEPS of complete graphs," Ars Mathematica Contemporanea, vol. 6, pp. 289299, 2013.

[34] M. Berhe Belay and C. Wang, "The first general Zagreb coindex of graph operations," Applied Mathematics and Nonlinear Sciences, vol. 5, no. 2, pp. 109-120, 2020.

[35] J.-B. Liu, J. Zhao, H. He, and Z. Shao, "Valency-based topological descriptors and structural property of the generalized sierpiński networks," Journal of Statistical Physics, vol. 177, no. 6, pp. 1131-1147, 2019.

[36] J.-B. Liu, J. Zhao, and Z.-Q. Cai, "On the generalized adjacency, laplacian and signless laplacian spectra of the weighted edge corona networks," Physica A: Statistical Mechanics and Its Applications, vol. 540, Article ID 123073, 2020.

[37] J.-B. Liu and X.-F. Pan, "Minimizing kirchhoff index among graphs with a given vertex bipartiteness," Applied Mathematics and Computation, vol. 291, pp. 84-88, 2016.

[38] D. Stevanović, "When is NEPS of graphs connected?" Linear Algebra and Its Applications, vol. 301, pp. 137-144, 1999.

[39] D. Stevanović, "When can the components of NEPS be almost cospectral?" Linear Algebra and Its Applications, vol. 311, pp. $35-44,2000$.

[40] D. Stevanović, "On the components of NEPS of connected bipartite graphs," Linear Algebra and Its Applications, vol. 356, pp. 67-78, 2002.

[41] D. Stevanoviz, "Energy and NEPS of graphs," Linear and Multilinear Algebra, vol. 53, no. 1, pp. 67-74, 2005.

[42] D. Cvetković, M. Doob, and H. Sachs, Spectra of Graphs Theory and Application, Johann Ambrosius Barth Verlag, Heidelberg, Leipzig, 1995. 